\title{
Triple rough statistical convergence of sequence of Bernstein operators
}

\author{
Ayten Esi ${ }^{1, *}$, N. Subramanian ${ }^{2}$, Ayhan Esi ${ }^{1}$ \\ ${ }^{1}$ Adiyaman University, Science and Arts Faculty, Department of Mathematics, 02040, Adiyaman, Turkey \\ ${ }^{2}$ Department of Mathematics, SASTRA University, Thanjavur-613 401, India
}

\section{A R T I C L E INFO}

Article history:

Received 24 November 2016

Received in revised form

26 January 2017

Accepted 26 January 2017

Keywords:

Rough statistical convergence

Natural density

Triple sequences

\begin{abstract}
A B S T R A C T
In this paper, using the concept of natural density, we introduce the notion of Bernstein operator of rough statistical convergence of triple sequence. We define the set of Bernstein operator of rough statistical limit points of a triple sequence spaces and obtain Bernstein operator of statistical convergence criteria associated with this set. Later, we prove that this set is closed and convex and also examine the relations between the set of Bernstein operator of rough statistical cluster points and the set of Bernstein operator of rough statistical limit points of a triple sequences.
\end{abstract}

(C) 2017 The Authors. Published by IASE. This is an open access article under the CC BY-NC-ND license (http://creativecommons.org/licenses/by-nc-nd/4.0/).

$K_{\varepsilon}:=\left\{(m, n, k) \in \mathbb{N}^{3}:\left|B_{m n k}(f, x)-f(x)\right| \geq \varepsilon\right\}$

\section{Introduction}

The idea of statistical convergence was introduced by Steinhaus (1951) and also independently by Fast (1951) for real or complex sequences. Statistical convergence is a generalization of the usual notion of convergence, which parallels the theory of ordinary convergence.

Let $K$ be a subset of the set of positive integers $\mathbb{N} \times \mathbb{N} \times \mathbb{N}$, and let us denote the set $\{(m, n, k) \in$ $K: m \leq u, n \leq v, k \leq w\}$ by $K_{u v w}$. Then the natural density of $K$ is given by $\delta(K)=\lim _{u v w \rightarrow \infty} \frac{\left|K_{u v w}\right|}{u v w}$, where $\left|K_{u v w}\right|$ denotes the number of elements in $K_{u v w}$. Clearly, a finite subset has natural density zero, and we have $\delta\left(K^{c}\right)=1-\delta(K)$ where $K^{c}=\mathbb{N} \backslash K$ is the complement of $K$. If $K_{1} \subseteq K_{2}$, then $\delta\left(K_{1}\right) \leq \delta\left(K_{2}\right)$.

The Bernstein operator of order $(r, s, t)$ is given by:

$B_{\text {rst }}(f, x)=$

$\sum_{m=0}^{r} \sum_{n=0}^{s} \sum_{k=0}^{t} f\left(\frac{m n k}{r s t}\right)\left(\begin{array}{l}r \\ m\end{array}\right)\left(\begin{array}{l}s \\ n\end{array}\right)\left(\begin{array}{l}t \\ k\end{array}\right) x^{m+n+k}(1-$

$x)^{(m-r)+(n-s)+(k-t)}$

where, $f$ is a continuous (real or complex valued) function defined on $[0,1]$.

Let $f$ be a continuous function defined on the closed interval $[0,1]$. A triple sequence of Bernstein polynomials $\left(B_{r s t}(f, x)\right)$ is said to be statistically convergent to $f(x)$, provided that the set:

\footnotetext{
* Corresponding Author.

Email Address: aesi23@hotmail.com (A.Esi)

https://doi.org/10.21833/ijaas.2017.02.005

2313-626X/C) 2017 The Authors. Published by IASE.

This is an open access article under the CC BY-NC-ND license

(http://creativecommons.org/licenses/by-nc-nd/4.0/)
}

has natural density zero for any $\varepsilon>0$. In this case, $f(x)$ is called the statistical limit of the triple sequence of Bernstein polynomials. i.e., $\delta\left(K_{\varepsilon}\right)=0$. That is,

$$
\begin{aligned}
& \lim _{r s t \rightarrow \infty} \frac{1}{r s t} \mid\left\{(m, n, k) \leq(r, s, t):\left|B_{m n k}(f, x)-f(x)\right| \geq\right. \\
& \varepsilon\} \mid=0 .
\end{aligned}
$$

In this case, we write $\delta-\lim B_{m n k}(f, x)=f(x)$ or $B_{m n k}(f, x) \rightarrow^{S_{B}} f(x)$.

If a triple sequence is statistically convergent, then for every $\varepsilon>0$, infinitely many terms of the sequence may remain outside the $\varepsilon-$ neighbourhood of the statistical limit, provided that the natural density of the set consisting of the indices of these terms is zero. This is an important property that distinguishes statistical convergence from ordinary convergence. Because the natural density of a finite set is zero, we can say that every ordinary convergent sequence is statistically convergent.

If a triple sequence satisfies some property $\mathrm{P}$ for all $m, n, k$ except a set of natural density zero, then we say that the triple sequence satisfies $P$ for almost all $(\mathrm{m}, \mathrm{n}, \mathrm{k})$ and we abbreviate this by a.a. $(\mathrm{m}, \mathrm{n}, \mathrm{k})$.

Let $\left(x_{m_{i} n_{j} k_{\ell}}\right)$ be a subsequence of $x=\left(x_{m n k}\right)$. If the natural density of the set $K=\left\{\left(m_{i}, n_{j}, k_{\ell}\right) \in\right.$ $\left.\mathbb{N}^{3}:(i, j, \ell) \in \mathbb{N}^{3}\right\}$ is different from zero, then $\left(x_{m_{i}} n_{j} k_{\ell}\right)$ is called a non thin subsequence of a triple sequence $\mathrm{x}$.

$c \in \mathbb{R}$ is called a statistical cluster point of a triple sequence $\mathrm{x}=\left(\mathrm{x}_{\mathrm{mnk}}\right)$ provided that the natural density of the set,

$\left\{(\mathrm{m}, \mathrm{n}, \mathrm{k}) \in \mathbb{N}^{3}:\left|\mathrm{x}_{\mathrm{mnk}}-\mathrm{c}\right|<\varepsilon\right\}$ 
is different from zero for every $\varepsilon>0$. We denote the set of all statistical cluster points of the sequence $x$ by $\Gamma_{\mathrm{x}}$.

Let $\mathrm{f}$ be a continuous function defined on the closed interval $[0,1]$. A triple sequence of Bernstein polynomials $\left(\mathrm{B}_{\mathrm{rst}}(\mathrm{f}, \mathrm{x})\right)$ is said to be statistically analytic if there exists a positive number $M$ such that,

$\delta\left(\left\{(\mathrm{m}, \mathrm{n}, \mathrm{k}) \in \mathbb{N}^{3}:\left|\mathrm{B}_{\mathrm{mnk}}(\mathrm{f}, \mathrm{x})-\mathrm{f}(\mathrm{x})\right|^{1 / \mathrm{m}+\mathrm{n}+\mathrm{k}} \geq \mathrm{M}\right\}\right)=0$

that is,

$$
\begin{gathered}
\lim _{r s t \rightarrow \infty} \frac{1}{r s t} \mid\left\{(m, n, k) \leq(r, s, t):\left|B_{m n k}(f, x)-f(x)\right|^{1 / m+n+k}\right. \\
\geq M\} \mid=0 .
\end{gathered}
$$

The theory of statistical convergence has been discussed in trigonometric series, summability theory, measure theory, turnpike theory, approximation theory, fuzzy set theory and so on.

The idea of rough convergence was introduced by Phu (2001), who also introduced the concepts of rough limit points and roughness degree. The idea of rough convergence occurs very naturally in numerical analysis and has interesting applications. Aytar (2008) extended the idea of rough convergence into rough statistical convergence using the notion of natural density just as usual convergence was extended to statistical convergence. Pal et al. (2013) extended the notion of rough convergence using the concept of ideals which automatically extends the earlier notions of rough convergence and rough statistical convergence.

In this paper, we introduce the notion of Bernstein operator of rough statistical convergence of triple sequences. Defining the set of Bernstein polynomials of rough statistical limit points of a triple sequence, we obtain to Bernstein operator of statistical convergence criteria associated with this set. Later, we prove that this set of Bernstein operator of statistical cluster points and the set of rough statistical limit points of a triple sequence.

A triple sequence (real or complex) can be defined as a function $\mathrm{x}: \mathbb{N} \times \mathbb{N} \times \mathbb{N} \rightarrow \mathbb{R}(\mathbb{C})$, where $\mathbb{N}, \mathbb{R}$ and $\mathbb{C}$ denote the set of natural numbers, real numbers and complex numbers respectively. The different types of notions of triple sequence was introduced and investigated at the initial by Sahiner et al. (2007), Sahiner and Tripathy (2008), Esi (2014), Esi and Catalbas (2014), Esi and Savas (2015), Esi et al. (2016), Dutta et al. (2013), Subramanian and Esi (2015), Debnath et al. (2015), Tripathy and Goswami $(2014,2015 a, b, c)$ and many others.

Throughout the paper let $r$ is a nonnegative real number.

\section{Definitions and preliminaries}

Definition 2.1: Let $f$ be a continuous function defined on the closed interval $[0,1]$. A triple sequence of Bernstein polynomials $\left(B_{m n k}(f, x)\right)$ is said to be $r-$ convergent to $f(x)$ denoted by $\mathrm{B}_{\mathrm{mnk}}(\mathrm{f}, \mathrm{x}) \rightarrow^{\mathrm{r}} \mathrm{f}(\mathrm{x})$, provided that,

$\forall \varepsilon>0 \quad \exists\left(\mathrm{m}_{\varepsilon}, \mathrm{n}_{\varepsilon}, \mathrm{k}_{\varepsilon}\right) \in \mathbb{N}^{3}: \mathrm{m} \geq \mathrm{m}_{\varepsilon}, \mathrm{n} \geq \mathrm{n}_{\varepsilon}, \mathrm{k} \geq \mathrm{k}_{\varepsilon}$ $\Rightarrow\left|\mathrm{B}_{\mathrm{mnk}}(\mathrm{f}, \mathrm{x})-\mathrm{f}(\mathrm{x})\right|<\mathrm{r}+\varepsilon$.

The set

$\operatorname{LIM}^{\mathrm{r}} \mathrm{B}_{\mathrm{mnk}}(\mathrm{f}, \mathrm{x})=\left\{\mathrm{L} \in \mathbb{R}^{3}: \mathrm{B}_{\mathrm{mnk}}(\mathrm{f}, \mathrm{x}) \rightarrow^{\mathrm{r}} \mathrm{f}(\mathrm{x})\right\}$

is called the $r$ - limit set of Bernstein operator of the triple sequences.

Definition 2.2: Let $f$ be a continuous function defined on the closed interval $[0,1]$. A triple sequence $\mathrm{x}=\left(\mathrm{x}_{\mathrm{mnk}}\right)$ of Bernstein polynomials $\left(B_{m n k}(f, x)\right)$ is said to be $r-$ convergent if $\operatorname{LIM}^{\mathrm{r}} \mathrm{B}_{\text {mnk }}(\mathrm{f}, \mathrm{x}) \neq \phi$. In this case, $\mathrm{r}$ is called the Bernstein operator of convergence degree of the triple sequence. For $r=0$, we get the ordinary convergence.

Definition 2.3: Let $f$ be a continuous function defined on the closed interval $[0,1]$. A triple sequence of Bernstein polynomials $\left(B_{m n k}(f, x)\right)$ is said to be $r-$ statistically convergent to $f(x)$, denoted by $B_{m n k}(f, x) \rightarrow^{r s t} f(x)$, provided that the set

$\left\{(\mathrm{m}, \mathrm{n}, \mathrm{k}) \in \mathbb{N}^{3}:\left|\mathrm{B}_{\mathrm{mnk}}(\mathrm{f}, \mathrm{x})-\mathrm{f}(\mathrm{x})\right| \geq \mathrm{r}+\varepsilon\right\}$

has natural density zero for every $\varepsilon>0$, or equivalently, if the condition,

st $-\lim \sup \left|\mathrm{B}_{\mathrm{mnk}}(\mathrm{f}, \mathrm{x})-\mathrm{f}(\mathrm{x})\right| \leq \mathrm{r}$

is satisfied.

In addition, we can write $B_{m n k}(f, x) \rightarrow^{\text {rst }} f(x)$ if and only if the inequality,

$\left|B_{\text {mnk }}(f, x)-f(x)\right|<r+\varepsilon$

holds for every $\varepsilon>0$ and almost all $(m, n, k)$. Here $r$ is called the Bernstein operator of roughness of degree. If we take $r=0$, then we obtain the ordinary statistical convergence of triple sequence.

In a similar fashion to the idea of classic rough convergence, the idea of Bernstein operator of rough statistical convergence of a triple sequence spaces can be interpreted as follows:

Assume that $\mathrm{f}$ be a continuous function defined on the closed interval [0,1]. A triple sequence of Bernstein polynomials $\left(B_{\mathrm{mnk}}(\mathrm{f}, \mathrm{y})\right)$ is statistically convergent and cannot be measured or calculated exactly; one has to do with an approximated (or statistically approximated) triple sequence of Bernstein polynomials $\left(\mathrm{B}_{\mathrm{mnk}}(\mathrm{f}, \mathrm{x})\right)$ satisfying $\left|B_{m n k}(f, x)-B_{m n k}(f, y)\right| \leq r$ for all $m, n, k$ (or for almost all $(m, n, k)$, i.e.,

$\delta\left(\left\{(\mathrm{m}, \mathrm{n}, \mathrm{k}) \in \mathbb{N}^{3}:\left|\mathrm{B}_{\mathrm{mnk}}(\mathrm{f}, \mathrm{x})-\mathrm{B}_{\mathrm{mnk}}(\mathrm{f}, \mathrm{y})\right|>\mathrm{r}\right\}\right)=0$.

Then the triple sequence of Bernstein polynomials $\left(\mathrm{B}_{\mathrm{mnk}}(\mathrm{f}, \mathrm{x})\right)$ is not statistically convergent any more, but as the inclusion, 
$\left\{(\mathrm{m}, \mathrm{n}, \mathrm{k}) \in \mathbb{N}^{3}:\left|\mathrm{B}_{\mathrm{mnk}}(\mathrm{f}, \mathrm{y})-\mathrm{f}(\mathrm{x})\right| \geq \varepsilon\right\} \supseteq\{(\mathrm{m}, \mathrm{n}, \mathrm{k}) \in$

$\left.\mathbb{N}^{3}:\left|B_{\text {mnk }}(f, x)-f(x)\right| \geq r+\varepsilon\right\}$

holds and we have:

$\delta\left(\left\{(\mathrm{m}, \mathrm{n}, \mathrm{k}) \in \mathbb{N}^{3}:\left|\mathrm{B}_{\mathrm{mnk}}(\mathrm{f}, \mathrm{y})-\mathrm{f}(\mathrm{x})\right| \geq \varepsilon\right\}\right)=0$,

i.e., we get,

$\delta\left(\left\{(\mathrm{m}, \mathrm{n}, \mathrm{k}) \in \mathbb{N}^{3}:\left|\mathrm{B}_{\mathrm{mnk}}(\mathrm{f}, \mathrm{x})-\mathrm{f}(\mathrm{x})\right| \geq \mathrm{r}+\varepsilon\right\}\right)=0$,

i.e., the triple sequence of Bernstein polynomials $\left(B_{\text {mnk }}(f, x)\right)$ is $r-$ statistically convergent in the sense of definition 2.3.

In general, the rough statistical limit of a triple sequence of Bernstein polynomials $\left(B_{m n k}(f, x)\right)$ may not unique for the roughness degree $r>0$. So we have to consider the so called $r$ - statistical limit set of a triple sequence of Bernstein polynomials $\left(B_{\text {mnk }}(f, x)\right)$, which is defined by:

st $-\operatorname{LIM}^{\mathrm{r}}\left(\mathrm{B}_{\mathrm{mnk}}(\mathrm{f}, \mathrm{x})\right)=\left\{\mathrm{L} \in \mathbb{R}: \mathrm{B}_{\mathrm{mnk}}(\mathrm{f}, \mathrm{x}) \rightarrow^{\mathrm{rst}} \mathrm{f}(\mathrm{x})\right\}$.

The triple sequence of Bernstein polynomials $\left(B_{\mathrm{mnk}}(\mathrm{f}, \mathrm{x})\right)$ is said to be $\mathrm{r}-$ statistically convergent provided that $s t-\operatorname{LIM}^{\mathrm{r}}\left(\mathrm{B}_{\mathrm{mnk}}(\mathrm{f}, \mathrm{x})\right) \neq \phi$. It is clear that if $s t-\operatorname{LIM}^{r}\left(B_{\text {mnk }}(f, x)\right) \neq \phi$. for a triple sequence of Bernstein polynomials $\left(B_{m n k}(f, x)\right)$ of real numbers, then we have:

st $-\operatorname{LIM}^{r}\left(B_{m n k}(f, x)\right)=\left[\right.$ st $-\lim \sup \left(B_{m n k}(f, x)\right)-$

$\left.r, s t-\lim \inf \left(B_{m n k}(f, x)\right)+r\right]$

We know that $\mathrm{LIM}^{\mathrm{r}}=\phi$ for an unbounded triple sequence of Bernstein polynomials $\left(B_{m n k}(f, x)\right)$. But such a triple sequence of Bernstein polynomials might be rough statistically convergent. For instance, define:

$\left(\mathrm{B}_{\mathrm{mnk}}(\mathrm{f}, \mathrm{x})\right)$

$= \begin{cases}(-1)^{m n k}, & \text { if }(m, n, k) \neq(i, j, \ell)^{2}(i, j, \ell \in \mathbb{N}), \\ (m n k), & \text { otherwise }\end{cases}$

in $\mathbb{R}$. Because the set $\{1,64,739, \cdots\}$ has natural density zero, we have

$s t-\operatorname{LIM}^{r}\left(B_{m n k}(f, x)\right)=\left\{\begin{array}{ll}\phi, & \text { if } r<1, \\ {[1-r, r-1],} & \text { otherwise }\end{array}\right\}$

and

$\operatorname{LIM}^{\mathrm{r}}\left(\mathrm{B}_{\mathrm{mnk}}(\mathrm{f}, \mathrm{x})\right)=\phi$ for all $\mathrm{r} \geq 0$

It can be seen from the above example fact that the fact $s t-\operatorname{LIM}^{r}\left(B_{\text {mnk }}(f, x)\right) \neq \phi$ does not imply $\operatorname{LIM}^{\mathrm{r}}\left(\mathrm{B}_{\mathrm{mnk}}(\mathrm{f}, \mathrm{x})\right) \neq \phi$. Because a finite set of natural numbers has natural density zero, $\operatorname{LIM}^{\mathrm{r}}\left(\mathrm{B}_{\mathrm{mnk}}(\mathrm{f}, \mathrm{x})\right) \neq \phi \quad$ implies st $\operatorname{LIM}^{\mathrm{r}}\left(\mathrm{B}_{\mathrm{mnk}}(\mathrm{f}, \mathrm{x})\right) \neq \phi$. Therefore, we get $\operatorname{LIM}^{\mathrm{r}}\left(\mathrm{B}_{\mathrm{mnk}}(\mathrm{f}, \mathrm{x})\right) \subseteq \mathrm{st}-\operatorname{LIM}^{\mathrm{r}}\left(\mathrm{B}_{\mathrm{mnk}}(\mathrm{f}, \mathrm{x})\right) . \quad$ This obvious fact means $\left\{r \geq 0: \operatorname{LIM}^{r}\left(B_{\text {mnk }}(f, x)\right) \neq \phi\right\} \subseteq$ $\left\{r \geq 0: s t-\operatorname{LIM}^{r}\left(B_{\text {mnk }}(f, x)\right) \neq \phi\right\}$ in this language of sets and yields immediately

$$
\begin{aligned}
\inf \left\{r \geq 0: \operatorname{LIM}^{r}\left(B_{\text {mnk }}(f, x)\right) \neq \phi\right\} & \\
& \geq \inf \left\{r \geq 0: s t-\operatorname{LIM}^{r}\left(B_{m n k}(f, x)\right) \neq \phi\right\} .
\end{aligned}
$$

Moreover, it also yields directly:

$\operatorname{diam}\left(\operatorname{LIM}^{r}\left(B_{m n k}(f, x)\right)\right) \leq \operatorname{diam}\left(\operatorname{st}-\operatorname{LIM}^{r}\left(B_{m n k}(f, x)\right)\right)$.

\section{Main results}

Theorem 3.1: Let $\mathrm{f}$ be a continuous function defined on the closed interval [0,1]. A triple sequence of Bernstein polynomials $\left(B_{m n k}(f, x)\right)$, we have $\operatorname{diam}\left(s t-\operatorname{LIM}^{r}\left(B_{m n k}(f, x)\right)\right) \leq 2 r$. In general $\operatorname{diam}\left(\mathrm{st}-\operatorname{LIM}^{\mathrm{r}}\left(\mathrm{B}_{\mathrm{mnk}}(\mathrm{f}, \mathrm{x})\right)\right)$ has an upper bound.

Proof: Assume that $\operatorname{diam}\left(\mathrm{st}-\operatorname{LIM}^{\mathrm{r}}\left(\mathrm{B}_{\mathrm{mnk}}(\mathrm{f}, \mathrm{x})\right)\right)>$ 2r. Then there exist $w, y \in s t-\operatorname{LIM}^{r}\left(B_{\text {rst }}(f, x)\right)$ such that $\left|\left(B_{\text {mnk }}(f, w)\right)-\left(B_{\text {mnk }}(f, y)\right)\right|>2$ r. Take $\varepsilon \in$ $\left(0, \frac{\left|\left(B_{m n k}(f, w)\right)-\left(B_{m n k}(f, y)\right)\right|}{2}-r\right)$. Because $\quad w, y \in$ st $\operatorname{LIM}^{\mathrm{r}}\left(\mathrm{B}_{\mathrm{mnk}}(\mathrm{f}, \mathrm{x})\right)$, we have $\delta\left(\mathrm{K}_{1}\right)=0$ and $\delta\left(\mathrm{K}_{2}\right)=0$ for every $\varepsilon>0$ where $\mathrm{K}_{1}=\{(\mathrm{m}, \mathrm{n}, \mathrm{k}) \in$ $\left.\mathbb{N}^{3}:\left|B_{\text {mnk }}(f, x)-w\right| \geq r+\varepsilon\right\} \quad$ and $\quad K_{2}=\{(m, n, k) \in$ $\left.\mathbb{N}^{3}:\left|B_{\text {mnk }}(f, x)-y\right| \geq r+\varepsilon\right\}$.

Using the properties of natural density, we get $\delta\left(\mathrm{K}_{1}^{\mathrm{c}} \cap \mathrm{K}_{2}^{\mathrm{c}}\right)=1$. Thus we can write:

$|\mathrm{w}-\mathrm{y}| \leq\left|\mathrm{B}_{\mathrm{mnk}}(\mathrm{f}, \mathrm{x})-\mathrm{w}\right|+\left|\mathrm{B}_{\mathrm{mnk}}(\mathrm{f}, \mathrm{x})-\mathrm{y}\right|$

$<2(r+\varepsilon)=2\left(\frac{|w-y|}{2}\right)=|w-y|$

for all $(m, n, k) \in K_{1}^{c} \cap \quad K_{2}^{c}$, which is a contradiction.

Now we prove the second part of the theorem. Consider a triple sequence of Bernstein polynomials $\left(B_{m n k}(f, x)\right)$ such that $s t-\lim B_{m n k}(f, x)=f(x)$. Let $\varepsilon>0$. Then we can write $\delta(\{(\mathrm{m}, \mathrm{n}, \mathrm{k}) \in$ $\left.\left.\mathbb{N}^{3}:\left|B_{\text {mnk }}(f, x)-f(x)\right| \geq \varepsilon\right\}\right)=0$. We have:

$$
\begin{aligned}
\left|B_{m n k}(f, x)-y\right| \leq & \left|B_{m n k}(f, x)-f(x)\right|+|f(x)-y| \\
& \leq\left|B_{m n k}(f, x)-f(x)\right|+r
\end{aligned}
$$

for each $y \in \bar{B}_{r}(f(x))=\left\{y \in \mathbb{R}^{3}:|y-f(x)| \leq r\right\}$.

Then we get $|f(x)-y|<r+\varepsilon$ for each $(m, n, k) \in$ $\left\{(\mathrm{m}, \mathrm{n}, \mathrm{k}) \in \mathbb{N}^{3}:\left|\mathrm{B}_{\mathrm{mnk}}(\mathrm{f}, \mathrm{x})-\mathrm{f}(\mathrm{x})\right|<\varepsilon\right\}$. Because the triple sequence space of Bernstein polynomials $\left(B_{\text {rst }}(f, x)\right)$ is statistically convergent to $f(x)$, we have

$\delta\left(\left\{(\mathrm{m}, \mathrm{n}, \mathrm{k}) \in \mathbb{N}^{3}:\left|\mathrm{B}_{\mathrm{mnk}}(\mathrm{f}, \mathrm{x})-\mathrm{f}(\mathrm{x})\right|<\varepsilon\right\}\right)=1$.

Therefore we get $y \in s t-\operatorname{LIM}^{r}\left(B_{r s t}(f, x)\right)$. Hence, we can write

$s t-\operatorname{LIM}^{r}\left(B_{r s t}(f, x)\right)=\bar{B}_{r}(f(x))$.

Because diam $\left(\bar{B}_{r}(f(x))\right)=2 r$, this shows that in general, the upper bound $2 r$ of the diameter of the set $s t-\operatorname{LIM}^{r}\left(B_{\text {rst }}(f, x)\right)$ is not a lower bound. 
Theorem 3.2: Let $\mathrm{f}$ be a continuous function defined on the closed interval $[0,1]$. A triple sequence of Bernstein polynomials $\left(\mathrm{B}_{\mathrm{mnk}}(\mathrm{f}, \mathrm{x})\right)$ is $\mathrm{r}$ - statistically convergent to $f(x)$ if and only if there exists a triple sequence of Bernstein polynomials $\left(B_{\text {rst }}(f, x)\right)$ such that $s t-\lim \left(B_{m n k}(f, y)\right)=f(x)$ and $\mid B_{m n k}(f, x)-$ $B_{\text {mnk }}(f, y) \mid \leq r$ for each $(m, n, k) \in \mathbb{N}^{3}$.

Proof. Necessity: Assume that $B_{m n k}(f, x) \rightarrow^{r s t} f(x)$. Then we have

st $-\lim \sup \left|B_{m n k}(f, x)-f(x)\right| \leq r$.

Now, define

$B_{m n k}(f, y)=\left(\begin{array}{l}f(x), \quad \text { if }\left|B_{m n k}(f, x)-f(x)\right| \leq r, \\ B_{m n k}(f, x)+r\left(\frac{f(x)-B_{m n k}(f, x)}{\left|B_{m n k}(f, x)-f(x)\right|}\right), \text { otherwise, }\end{array}\right.$

then, we write

$\left|B_{\text {mnk }}(f, y)-f(x)\right|=$

$\left(|f(x)-f(x)|\right.$, if $\left|B_{m n k}(f, x)-f(x)\right| \leq r$,

$\left(\left|B_{m n k}(f, x)-f(x)\right|+r\left(\frac{|f(x)-f(x)|-\left|B_{m n k}(f, x)-f(x)\right|}{\left|B_{m n k}(f, x)-f(x)\right|}\right)\right.$, otherwise, (i. e) $\left|B_{m n k}(f, y)-f(x)\right|=$

$\left(0\right.$, if $\left|B_{m n k}(f, x)-f(x)\right| \leq r$,

$\left(\left|B_{m n k}(f, x)-f(x)\right|-r\left(\frac{\left|B_{m n k}(f, x)-f(x)\right|}{\left|B_{m n k}(f, x)-f(x)\right|}\right), \quad\right.$ otherwise,

(i.e) $\left|B_{m n k}(f, y)-f(x)\right|$

$$
=\left(\begin{array}{l}
0, \quad \text { if }\left|B_{m n k}(f, x)-f(x)\right| \leq r \\
\left|B_{m n k}(f, x)-f(x)\right|-r, \quad \text { otherwise. }
\end{array}\right.
$$

We have

$\left|B_{m n k}(f, y)-f(x)\right| \geq\left|B_{m n k}(f, x)-f(x)\right|-r$

$\Rightarrow\left|\mathrm{B}_{\mathrm{mnk}}(\mathrm{f}, \mathrm{x})-\mathrm{f}(\mathrm{x})-\mathrm{B}_{\mathrm{mnk}}(\mathrm{f}, \mathrm{y})+\mathrm{f}(\mathrm{x})\right| \leq \mathrm{r}$

$\Rightarrow\left|B_{m n k}(f, x)-B_{m n k}(f, y)\right| \leq r$

for all $m, n, k \in \mathbb{N}^{3}$. By equation (3.1) and by definition of $\mathrm{y}_{\mathrm{mnk}}$, we get

st $-\limsup \left|B_{m n k}(f, y)-f(x)\right|=0$

$\Rightarrow$ st $-\lim B_{\mathrm{mnk}}(\mathrm{f}, \mathrm{y}) \rightarrow^{\mathrm{r}} \mathrm{f}(\mathrm{x})$.

Sufficiency: Since st $-\lim B_{m n k}(f, y)=f(x)$, we have

$$
\delta\left(\left\{(\mathrm{m}, \mathrm{n}, \mathrm{k}) \in \mathbb{N}^{3}:\left|\mathrm{B}_{\mathrm{mnk}}(\mathrm{f}, \mathrm{y})-\mathrm{f}(\mathrm{x})\right| \geq \varepsilon\right\}\right)=0
$$

for each $\varepsilon>0$. It is easy to see that the inclusion

$$
\begin{aligned}
\left\{(\mathrm{m}, \mathrm{n}, \mathrm{k}) \in \mathbb{N}^{3}:\left|\mathrm{B}_{\mathrm{mnk}}(\mathrm{f}, \mathrm{y})-\mathrm{f}(\mathrm{x})\right| \geq \varepsilon\right\} \\
\\
\supseteq\left\{(\mathrm{m}, \mathrm{n}, \mathrm{k}) \in \mathbb{N}^{3}:\left|\mathrm{B}_{\mathrm{mnk}}(\mathrm{f}, \mathrm{x})-\mathrm{f}(\mathrm{x})\right|\right. \\
\geq \mathrm{r}+\varepsilon\}
\end{aligned}
$$

holds.

Since

$\delta\left(\left\{(\mathrm{m}, \mathrm{n}, \mathrm{k}) \in \mathbb{N}^{3}:\left|\mathrm{y}_{\mathrm{mnk}}-\mathrm{f}(\mathrm{x})\right| \geq \varepsilon\right\}\right)=0$,

we get

$\delta\left(\left\{(\mathrm{m}, \mathrm{n}, \mathrm{k}) \in \mathbb{N}^{3}:\left|\mathrm{B}_{\mathrm{mnk}}(\mathrm{f}, \mathrm{x})-\mathrm{f}(\mathrm{x})\right| \geq \mathrm{r}+\varepsilon\right\}\right)=0$.
Remark 3.3: If we replace the condition $\left|B_{\text {mnk }}(f, x)-B_{\text {mnk }}(f, y)\right| \leq r$ for all $m, n, k \in \mathbb{N}^{3}$ in the hypothesis of the theorem 3.2 with the condition

$\delta\left(\left\{(\mathrm{m}, \mathrm{n}, \mathrm{k}) \in \mathbb{N}^{3}:\left|\mathrm{B}_{\mathrm{mnk}}(\mathrm{f}, \mathrm{x})-\mathrm{B}_{\mathrm{mnk}}(\mathrm{f}, \mathrm{y})\right|>\mathrm{r}\right\}\right)=0$

is valid.

Theorem 3.4: Let $\mathrm{f}$ be a continuous function defined on the closed interval $[0,1]$. A triple sequence of Bernstein polynomials $\left(\mathrm{B}_{\mathrm{mnk}}(\mathrm{f}, \mathrm{x})\right)$ is an arbitrary $c \in \Gamma_{\mathrm{x}}$ of triple sequence of Bernstein polynomials $\left(B_{\text {mnk }}(f, x)\right)$ we have $|f(x)-c| \leq r$ for all $f(x) \in$ st $\operatorname{LIM}^{\mathrm{r}}\left(\mathrm{B}_{\mathrm{mnk}}(\mathrm{f}, \mathrm{x})\right)$.

Proof: Assume on the contrary that there exist a point $c \in \Gamma_{\mathrm{x}}$ and $\mathrm{f}(\mathrm{x}) \in \mathrm{st}-\operatorname{LIM}^{\mathrm{r}}\left(\mathrm{B}_{\mathrm{mnk}}(\mathrm{f}, \mathrm{x})\right)$ such that $|f(x)-c|>r$. Define $\varepsilon:=\frac{|l-c|-r}{3}$. Then

$\left\{(\mathrm{m}, \mathrm{n}, \mathrm{k}) \in \mathbb{N}^{3}:|\mathrm{f}(\mathrm{x})-\mathrm{c}|<\varepsilon\right\} \subseteq\{(\mathrm{m}, \mathrm{n}, \mathrm{k}) \in$

$\left.\mathbb{N}^{3}:\left|\left(B_{\text {mnk }}(f, x)\right)-f(x)\right| \geq r+\varepsilon\right\}$.

Since $c \in \Gamma_{x}$, we have

$\delta\left(\left\{(\mathrm{m}, \mathrm{n}, \mathrm{k}) \in \mathbb{N}^{3}:\left|\left(\mathrm{B}_{\mathrm{mnk}}(\mathrm{f}, \mathrm{x})\right)-\mathrm{c}\right|<\varepsilon\right\}\right) \neq 0$.

Hence, by (3.3), we get

$\delta\left(\left\{(\mathrm{m}, \mathrm{n}, \mathrm{k}) \in \mathbb{N}^{3}:\left|\left(\mathrm{B}_{\mathrm{mnk}}(\mathrm{f}, \mathrm{x})\right)-\mathrm{f}(\mathrm{x})\right| \geq \mathrm{r}+\varepsilon\right\}\right) \neq 0$,

which contradicts the fact $l \in s t-\operatorname{LIM}^{\mathrm{r}}\left(\mathrm{B}_{\mathrm{mnk}}(\mathrm{f}, \mathrm{x})\right)$.

Proposition 3.5: Let $f$ be a continuous function defined on the closed interval $[0,1]$. A triple sequence of Bernstein polynomials $\left(B_{m n k}(f, x)\right)$ is analytic, then there exists a non-negative real number $r$ such that $s t-\operatorname{LIM}^{\mathrm{r}}\left(\mathrm{B}_{\mathrm{mnk}}(\mathrm{f}, \mathrm{x})\right) \neq \phi$.

Proof: If we take the triple sequence of Bernstein polynomials $\left(\mathrm{B}_{\mathrm{mnk}}(\mathrm{f}, \mathrm{x})\right)$ is to be statistically analytic, then the of proposition holds. Thus we have the following theorem.

Theorem 3.6: Let $\mathrm{f}$ be a continuous function defined on the closed interval $[0,1]$. A triple sequence of Bernstein polynomials $\left(B_{\mathrm{mnk}}(\mathrm{f}, \mathrm{x})\right)$ is statistically analytic if and only if there exists a non-negative real number $r$ such that $s t-\operatorname{LIM}^{\mathrm{r}}\left(\mathrm{B}_{\mathrm{mnk}}(\mathrm{f}, \mathrm{x})\right) \neq \phi$.

Proof: Since the triple sequence of Bernstein polynomials $\left(B_{\mathrm{mnk}}(\mathrm{f}, \mathrm{x})\right)$ is statistically analytic, there exists a positive real number $M$ such that

$\delta\left(\left\{(\mathrm{m}, \mathrm{n}, \mathrm{k}) \in \mathbb{N}^{3}:\left|\left(\mathrm{B}_{\mathrm{mnk}}(\mathrm{f}, \mathrm{x})\right)\right|^{1 / \mathrm{m}+\mathrm{n}+\mathrm{k}} \geq \mathrm{M}\right\}\right)=0$.

Define

$r^{\prime}=\sup \left\{\left|\left(B_{m n k}(f, x)\right)\right|^{1 / m+n+k}:(m, n, k) \in K^{c}\right\}$,

where

$K=\left\{(m, n, k) \in \mathbb{N}^{3}:\left|\left(B_{m n k}(f, x)\right)\right|^{1 / m+n+k} \geq M\right\}$ 
Then the set st $-\operatorname{LIM}^{\mathrm{r}}\left(\mathrm{B}_{\mathrm{mnk}}(\mathrm{f}, \mathrm{x})\right)$ contains the origin of $\mathbb{R}$. So we have st $-\operatorname{LIM}^{r}\left(B_{\text {mnk }}(f, x)\right) \neq \phi$.

If st $-\operatorname{LIM}^{r}\left(B_{\text {mnk }}(f, x)\right) \neq \phi$ for some $r \geq 0$, then there exists $f(x)$ such that $f(x) \in$ st $\operatorname{LIM}^{r}\left(B_{m n k}(f, x)\right)$, i.e.,

$$
\begin{gathered}
\delta\left(\left\{(\mathrm{m}, \mathrm{n}, \mathrm{k}) \in \mathbb{N}^{3}:\left|\left(\mathrm{B}_{\mathrm{mnk}}(\mathrm{f}, \mathrm{x})\right)-\mathrm{f}(\mathrm{x})\right|^{1 / \mathrm{m}+\mathrm{n}+\mathrm{k}} \geq \mathrm{r}+\varepsilon\right\}\right) \\
=0
\end{gathered}
$$

for each $\varepsilon>0$.

Then we say that almost all triple sequence are contained in some ball with any radius greater than $r$. So the triple sequence space of Bernstein polynomials $\left(B_{\mathrm{mnk}}(\mathrm{f}, \mathrm{x})\right)$ is statistically analytic.

Remark 3.7: If $x^{\prime}=\left(x_{m_{i}, n_{j}, k_{\ell}}\right)$ is a sub sequence of $\mathrm{x}=\left(\mathrm{x}_{\mathrm{mnk}}\right), \quad$ then $\operatorname{LIM}^{\mathrm{r}}\left(\mathrm{B}_{\text {mnk }}(\mathrm{f}, \mathrm{x})\right) \subseteq$ $\operatorname{LIM}^{\mathrm{r}}\left(\mathrm{B}_{\mathrm{mnk}}(\mathrm{f}, \mathrm{x})\right)^{\prime}$. But it is not valid for statistical convergence.

Example 3.8: Define

$$
\begin{aligned}
& \left(B_{m n k}(f, x)\right) \\
& =\left\{\begin{array}{ll}
(m n k), & \text { if }(m, n, k)=(i, j, \ell)^{2}(i, j, \ell \in \mathbb{N}), \\
0, & \text { otherwise }
\end{array}\right\}
\end{aligned}
$$

of real numbers. Then the triple sequence spaces $\left(B_{m n k}(f, x)\right)^{\prime}=(1,64,739, \cdots)$ is a subsequence of $\left(B_{\text {mnk }}(f, x)\right)$. We have st $-\operatorname{LIM}^{r}\left(B_{\text {mnk }}(f, x)\right)=[-r, r]$ abd st $-\operatorname{LIM}^{\mathrm{r}}\left(\mathrm{B}_{\mathrm{mnk}}(\mathrm{f}, \mathrm{x})\right)^{\prime}=\phi$.

Theorem 3.9: Let $\mathrm{x}^{\prime}=\left(\mathrm{x}_{\left.\mathrm{m}_{\mathrm{i}}, \mathrm{n}_{\mathrm{j}}, \mathrm{k}_{\ell}\right)}\right)$ is a non thin sub sequence of triple sequence space of Bernstein polynomials $\left(B_{\mathrm{mnk}}(\mathrm{f}, \mathrm{x})\right)$, then st $\operatorname{LIM}^{\mathrm{r}},\left(\mathrm{B}_{\mathrm{mnk}}(\mathrm{f}, \mathrm{x})\right) \subseteq \mathrm{st}-\operatorname{LIM}^{\mathrm{r}}\left(\mathrm{B}_{\mathrm{mnk}}(\mathrm{f}, \mathrm{x})\right)^{\prime}$.

Proof: Omitted.

Theorem 3.10: Let $\mathrm{f}$ be a continuous function defined on the closed interval $[0,1]$. A triple sequence of Bernstein polynomials $\left(B_{m n k}(f, x)\right)$ of $r$ - statistical limit set is closed.

Proof: If $s t-\operatorname{LIM}^{r}\left(B_{m n k}(f, x)\right) \neq \phi$, then it is true. Assume that st $-\operatorname{LIM}^{\mathrm{r}}\left(\mathrm{B}_{\mathrm{mnk}}(\mathrm{f}, \mathrm{x})\right) \neq \phi$, then we can choose a triple sequence space of Bernstein polynomials $\quad\left(B_{m n k}(f, y)\right) \subseteq s t-\operatorname{LIM}^{r}\left(B_{m n k}(f, x)\right)$ such that $\left(B_{m n k}(f, y)\right) \rightarrow{ }^{r} f(x)$ as $m, n, k \rightarrow \infty$. If we prove that $f(x) \in s t-\operatorname{LIM}^{r}\left(B_{m n k}(f, x)\right)$, then the proof will be complete.

Let $\varepsilon>0$ be given. Since $\left(\mathrm{B}_{\text {mnk }}(\mathrm{f}, \mathrm{y})\right) \rightarrow^{\mathrm{r}} \mathrm{f}(\mathrm{x}), \exists\left(\mathrm{m}_{\frac{\varepsilon}{2}}, \mathrm{n}_{\frac{\varepsilon}{2}}, \mathrm{k}_{\frac{\varepsilon}{2}}\right) \in \mathbb{N}^{3}$ such that

$$
\left|\left(B_{m n k}(f, y)\right)-f(x)\right|<\frac{\varepsilon}{2} \text { for all } m>m_{\frac{\varepsilon}{2}}, n>n_{\frac{\varepsilon}{2}}, k>k_{\frac{\varepsilon}{2}}
$$

Now choose an $\left(\mathrm{m}_{0}, \mathrm{n}_{0} \mathrm{k}_{0}\right) \in \mathbb{N}^{3}$ such that $\mathrm{m}_{0}>$ $\mathrm{m}_{\frac{\varepsilon}{2}}, \mathrm{n}_{0}>\mathrm{n}_{\frac{\varepsilon}{2}}, \mathrm{k}_{0}>\mathrm{k}_{\frac{\varepsilon}{2}}$. Then we can write

$$
\left|\mathrm{B}_{\mathrm{m}_{0} \mathrm{n}_{0} \mathrm{k}_{0}}(\mathrm{f}, \mathrm{y})-\mathrm{f}(\mathrm{x})\right|<\frac{\varepsilon}{2}
$$

On the other hand, since $\left(B_{\mathrm{mnk}}(\mathrm{f}, \mathrm{y})\right) \subseteq \mathrm{st}-$

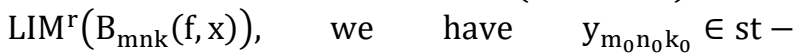
$\operatorname{LIM}^{\mathrm{r}}\left(\mathrm{B}_{\mathrm{mnk}}(\mathrm{f}, \mathrm{x})\right)$, namely,

$\delta\left(\left\{(\mathrm{m}, \mathrm{n}, \mathrm{k}) \in \mathbb{N}^{3}:\left|\left(\mathrm{B}_{\mathrm{mnk}}(\mathrm{f}, \mathrm{x})\right)-\mathrm{y}_{\mathrm{m}_{0} \mathrm{n}_{0} \mathrm{k}_{0}}\right| \geq \mathrm{r}+\frac{\varepsilon}{2}\right\}\right)=0$.

Now let us show that the inclusion

$\left\{(\mathrm{m}, \mathrm{n}, \mathrm{k}) \in \mathbb{N}^{3}:\left|\left(\mathrm{B}_{\mathrm{mnk}}(\mathrm{f}, \mathrm{x})\right)-\mathrm{f}(\mathrm{x})\right|<\mathrm{r}+\varepsilon\right\} \supseteq$

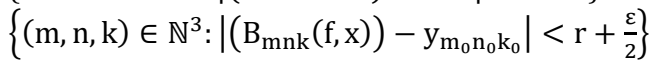

holds.

Take $\quad(\mathrm{i}, \mathrm{j}, \ell) \in\left\{(\mathrm{m}, \mathrm{n}, \mathrm{k}) \in \mathbb{N}^{3}: \mid\left(\mathrm{B}_{\mathrm{mnk}}(\mathrm{f}, \mathrm{x})\right)-\right.$ $\left.\mathrm{y}_{\mathrm{m}_{0} \mathrm{n}_{0} \mathrm{k}_{0}} \mid<\mathrm{r}+\frac{\varepsilon}{2}\right\}$. Then we have

$\left|\left(B_{m n k}(f, x)\right)-y_{m_{0} n_{0} k_{0}}\right|<r+\frac{\varepsilon}{2}$

and hence

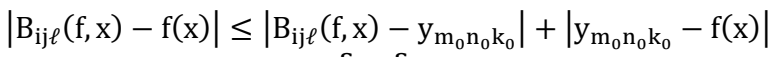

$$
\begin{aligned}
& <\mathrm{r}+\frac{\varepsilon}{2}+\frac{\varepsilon}{2}<\mathrm{r}+\varepsilon
\end{aligned}
$$

i.e., $\quad(i, j, \ell) \in\left\{(m, n, k) \in \mathbb{N}^{3}:\left|\left(B_{\text {mnk }}(f, x)\right)-f(x)\right|<\right.$ $r+\varepsilon\}$ which proves the equation (3.5). Hence the natural density of the set on the LHS of equation (3.5) is equal to 1 . So we get $\delta(\{(m, n, k) \in$ $\left.\left.\mathbb{N}^{3}:\left|\left(B_{\text {mnk }}(f, x)\right)-f(x)\right| \geq r+\varepsilon\right\}\right)=0$.

Theorem 3.11: Let $f$ be a continuous function defined on the closed interval $[0,1]$. A triple sequence of Bernstein polynomials $\left(B_{\text {mnk }}(f, x)\right)$ of $r$ - statistical limit set is convex.

Proof: Let $\mathrm{y}_{1}, \mathrm{y}_{2} \in \mathrm{st}-\operatorname{LIM}^{\mathrm{r}}\left(\mathrm{B}_{\mathrm{mnk}}(\mathrm{f}, \mathrm{x})\right)$ for the triple sequence of Bernstein polynomials $\left(\mathrm{B}_{\mathrm{mnk}}(\mathrm{f}, \mathrm{x})\right)$ and let $\varepsilon>0$ be given. Define $\mathrm{K}_{1}=$ $\left\{(\mathrm{m}, \mathrm{n}, \mathrm{k}) \in \mathbb{N}^{3}:\left|\left(\mathrm{B}_{\mathrm{mnk}}(\mathrm{f}, \mathrm{x})\right)-\mathrm{y}_{1}\right| \geq \mathrm{r}+\varepsilon\right\}$ and $\mathrm{K}_{2}=$ $\left\{(\mathrm{m}, \mathrm{n}, \mathrm{k}) \in \mathbb{N}^{3}:\left|\left(\mathrm{B}_{\mathrm{mnk}}(\mathrm{f}, \mathrm{x})\right)-\mathrm{y}_{2}\right| \geq \mathrm{r}+\varepsilon\right\}$. Because $\mathrm{y}_{1}, \mathrm{y}_{2} \in \mathrm{st}-\operatorname{LIM}^{\mathrm{r}}\left(\mathrm{B}_{\mathrm{mnk}}(\mathrm{f}, \mathrm{x})\right)$, we have $\delta\left(\mathrm{K}_{1}\right)=$ $\delta\left(\mathrm{K}_{2}\right)=0$. Thus we have

$$
\begin{aligned}
\mid\left(B_{\mathrm{mnk}}(\mathrm{f}, \mathrm{x})\right)-[(1 & \left.-\lambda) \mathrm{y}_{1}+\lambda \mathrm{y}_{2}\right] \mid \\
& =\mid(1-\lambda)\left(\left(\mathrm{B}_{\mathrm{mnk}}(\mathrm{f}, \mathrm{x})\right)-\mathrm{y}_{1}\right) \\
& +\lambda\left(\left(\mathrm{B}_{\mathrm{mnk}}(\mathrm{f}, \mathrm{x})\right)-\mathrm{y}_{2}\right) \mid<\mathrm{r}+\varepsilon,
\end{aligned}
$$

for each $(m, n, k) \in\left(K_{1}^{c} \cap K_{2}^{c}\right)$ and each $\lambda \in[0,1]$. Because $\delta\left(\mathrm{K}_{1}^{\mathrm{c}} \cap \mathrm{K}_{2}^{\mathrm{c}}\right)=1$, we get

$$
\begin{gathered}
\delta\left(\left\{(\mathrm{m}, \mathrm{n}, \mathrm{k}) \in \mathbb{N}^{3}:\left|\left(\mathrm{B}_{\mathrm{mnk}}(\mathrm{f}, \mathrm{x})\right)-\left[(1-\lambda) \mathrm{y}_{1}+\lambda \mathrm{y}_{2}\right]\right|\right.\right. \\
\geq \mathrm{r}+\varepsilon\})=0,
\end{gathered}
$$

i.e., $\left[(1-\lambda) y_{1}+\lambda y_{2}\right] \in s t-\operatorname{LIM}^{r}\left(B_{\text {mnk }}(f, x)\right)$, which proves the convexity of the set $s t-\operatorname{LIM}^{\mathrm{r}}\left(\mathrm{B}_{\mathrm{mnk}}(\mathrm{f}, \mathrm{x})\right)$. Theorem 3.12: Let $f$ be a continuous function defined on the closed interval $[0,1]$. A triple sequence of Bernstein polynomials $\left(B_{\mathrm{mnk}}(\mathrm{f}, \mathrm{x})\right)$ of statistically converges to $f(x)$ if and only if st $\operatorname{LIM}^{\mathrm{r}}\left(\mathrm{B}_{\mathrm{mnk}}(\mathrm{f}, \mathrm{x})\right)=\overline{\mathrm{B}}_{\mathrm{r}}(\mathrm{f}(\mathrm{x}))$. 
Proof: We have proved the necessity part of this theorem in proof of the theorem 3.1.

Sufficiency: Because $\quad s t-\operatorname{LIM}^{\mathrm{r}}\left(\mathrm{B}_{\mathrm{mnk}}(\mathrm{f}, \mathrm{x})\right)=$ $\overline{\mathrm{B}}_{\mathrm{r}}(\mathrm{f}(\mathrm{x})) \neq \phi$, then by Proposition (3.1) we can say that the triple sequence space of Bernstein polynomials $\left(\mathrm{B}_{\mathrm{mnk}}(\mathrm{f}, \mathrm{x})\right)$ is statistically analytic. Assume on the contrary that the triple sequence space of Bernstein polynomials $\left(\mathrm{B}_{\mathrm{mnk}}(\mathrm{f}, \mathrm{x})\right)$ has another statistical cluster point $\mathrm{f}^{\prime}(\mathrm{x})$ different from $\mathrm{f}(\mathrm{x})$. Then the point

$$
\bar{f}(x)=f(x)+\frac{r}{\left|f(x)-f^{\prime}(x)\right|}\left(f(x)-f^{\prime}(x)\right)
$$

satisfies

$$
\begin{aligned}
& \overline{\mathrm{f}}(\mathrm{x})-\mathrm{f}^{\prime}(\mathrm{x})=\mathrm{f}(\mathrm{x})-\mathrm{f}^{\prime}(\mathrm{x})+\frac{\mathrm{r}}{\left|\mathrm{f}(\mathrm{x})-\mathrm{f}^{\prime}(\mathrm{x})\right|}\left(\mathrm{f}(\mathrm{x})-\mathrm{f}^{\prime}(\mathrm{x})\right) \\
& \begin{aligned}
&\left|\overline{\mathrm{f}}(\mathrm{x})-\mathrm{f}^{\prime}(\mathrm{x})\right|=\left|\mathrm{f}(\mathrm{x})-\mathrm{f}^{\prime}(\mathrm{x})\right| \\
&+\frac{\mathrm{r}}{\left|\mathrm{f}(\mathrm{x})-\mathrm{f}^{\prime}(\mathrm{x})\right|}\left(\mathrm{f}(\mathrm{x})-\mathrm{f}^{\prime}(\mathrm{x})\right)
\end{aligned} \\
& \left|\overline{\mathrm{f}}(\mathrm{x})-\mathrm{f}^{\prime}(\mathrm{x})\right|=\left|\mathrm{f}(\mathrm{x})-\mathrm{f}^{\prime}(\mathrm{x})\right|+\mathrm{r}>\mathrm{r} .
\end{aligned}
$$

Since $\mathrm{f}^{\prime}(\mathrm{x})$ is a statistical cluster point of the triple sequence space of Bernstein polynomials $\left(B_{m n k}(f, x)\right)$, by theorem 3.4 this inequality implies that $\overline{\mathrm{f}}(\mathrm{x}) \notin \mathrm{st}-\operatorname{LIM}^{\mathrm{r}}\left(\mathrm{B}_{\mathrm{mnk}}(\mathrm{f}, \mathrm{x})\right)$. This contradicts the fact $|\bar{f}(\mathrm{x})-\mathrm{f}(\mathrm{x})|=\mathrm{r}$ and $\mathrm{st}-\operatorname{LIM}^{\mathrm{r}}\left(\mathrm{B}_{\mathrm{mnk}}(\mathrm{f}, \mathrm{x})\right)=$ $\bar{B}_{r}(f(x))$. Therefore, $f(x)$ is the unique statistical cluster point of the triple sequence space of Bernstein polynomials $\left(B_{m n k}(f, x)\right)$. Hence the statistical cluster point of a statistically analytic triple sequence space of Bernstein polynomials is unique, then the triple sequence space of Bernstein polynomials $\left(B_{m n k}(f, x)\right)$ is statistically convergent to $f(x)$.

Theorem 3.13: Let $\mathrm{f}$ be a continuous function defined on the closed interval $[0,1]$. A triple sequence of Bernstein polynomials $\left(B_{m n k}(f, x)\right)$ and $\left(\mathbb{R}^{3},|.|,\right)$ be a strictly convex space, there exist $\mathrm{y}_{1}, \mathrm{y}_{2} \in \mathrm{st}-\operatorname{LIM}^{\mathrm{r}}\left(\mathrm{B}_{\mathrm{mnk}}(\mathrm{f}, \mathrm{x})\right)$ such that $\left|\mathrm{y}_{1}-\mathrm{y}_{2}\right|=$ $2 r$ then this triple sequence of Bernstein polynomials $\left(B_{m n k}(f, x)\right)$ is statistically convergent to $\frac{1}{2}\left(y_{1}+y_{2}\right)$.

Proof: Assume that $\mathrm{z} \in \Gamma_{\mathrm{x}}$. Then $\mathrm{y}_{1}, \mathrm{y}_{2} \in \mathrm{st}-$ $\operatorname{LIM}^{\mathrm{r}}\left(\mathrm{B}_{\mathrm{mnk}}(\mathrm{f}, \mathrm{x})\right)$ implies that

$\left|\mathrm{y}_{1}-\mathrm{z}\right| \leq \mathrm{r}$ and $\left|\mathrm{y}_{2}-\mathrm{z}\right| \leq \mathrm{r}$,

by theorem 3.4. On the other hand, we have

$2 \mathrm{r}=\left|\mathrm{y}_{1}-\mathrm{y}_{2}\right| \leq\left|\mathrm{y}_{1}-\mathrm{z}\right|+\left|\mathrm{y}_{2}-\mathrm{z}\right|$

combining the inequalities (3.6) and (3.7), we get $\left|y_{1}-z\right|=\left|y_{2}-z\right|=r$. Since

$\frac{1}{2}\left(\mathrm{y}_{2}-\mathrm{y}_{1}\right)=\frac{1}{2}\left[\left(\mathrm{z}-\mathrm{y}_{1}\right)+\left(-\mathrm{z}+\mathrm{y}_{2}\right)\right]$

and $\left|y_{1}-y_{2}\right|=2 r$, we get $\left|\frac{1}{2}\left(y_{2}-y_{1}\right)\right|=r$. By the strict convexity of the space and from the equality
(3.8), we get $\frac{1}{2}\left(\mathrm{y}_{2}-\mathrm{y}_{1}\right)=\left(\mathrm{z}-\mathrm{y}_{1}\right)=\left(-\mathrm{z}+\mathrm{y}_{2}\right)$ which implies that $\mathrm{z}=\frac{1}{2}\left(\mathrm{y}_{1}+\mathrm{y}_{2}\right)$. Hence $\mathrm{z}$ is the unique statistical cluster point of the triple sequence space of Bernstein polynomials $\left(\mathrm{B}_{\mathrm{mnk}}(\mathrm{f}, \mathrm{x})\right)$.

On the other hand, the assumption $\mathrm{y}_{1}, \mathrm{y}_{2} \in \mathrm{st}-$ $\operatorname{LIM}^{\mathrm{r}} \mathrm{x}$ implies that $\mathrm{st}-\operatorname{LIM}^{\mathrm{r}}\left(\mathrm{B}_{\text {mnk }}(\mathrm{f}, \mathrm{x})\right) \neq 0$. By theorem 3.6, the triple sequence of Bernstein polynomials $\left(\mathrm{B}_{\mathrm{mnk}}(\mathrm{f}, \mathrm{x})\right)$ is statistically analytic. Consequently, the statistical cluster point of a statistically analytic triple sequence space of Bernstein polynomials $\left(\mathrm{B}_{\mathrm{mnk}}(\mathrm{f}, \mathrm{x})\right)$ is unique, then the triple sequence space of Bernstein polynomials $\left(B_{\text {mnk }}(f, x)\right)$ is statistically convergent, i.e.,

$s t-\lim \left(B_{\mathrm{mnk}}(\mathrm{f}, \mathrm{x})\right)=\frac{1}{2}\left(\mathrm{y}_{1}+\mathrm{y}_{2}\right)$.

Theorem 3.14: Let $f$ be a continuous function defined on the closed interval $[0,1]$. A triple sequence of Bernstein polynomials $\left(B_{\mathrm{mnk}}(\mathrm{f}, \mathrm{x})\right)$.

(a) If $c \in \Gamma_{x}$ then

st $-\operatorname{LIM}^{\mathrm{r}}\left(\mathrm{B}_{\mathrm{mnk}}(\mathrm{f}, \mathrm{x})\right) \subseteq \overline{\mathrm{B}}_{\mathrm{r}}(\mathrm{c})$

(b)st $-\operatorname{LIM}^{\mathrm{r}}\left(\mathrm{B}_{\mathrm{mnk}}(\mathrm{f}, \mathrm{x})\right)=\bigcap_{\mathrm{c} \in \Gamma_{\mathrm{x}}} \overline{\mathrm{B}}_{\mathrm{r}}(\mathrm{c})=\left\{\mathrm{f}(\mathrm{x}) \in \mathbb{R}^{3}: \Gamma_{\mathrm{x}} \subseteq\right.$ $\left.\overline{\mathrm{B}}_{\mathrm{r}}(\mathrm{f}(\mathrm{x}))\right\}$

Proof:

(a) Assume that $\mathrm{l} \in \mathrm{st}-\operatorname{LIM}^{\mathrm{r}}\left(\mathrm{B}_{\mathrm{mnk}}(\mathrm{f}, \mathrm{x})\right)$ and $\mathrm{c} \in \Gamma_{\mathrm{x}}$. Then by theorem 3.4, we have

$|\mathrm{f}(\mathrm{x})-\mathrm{c}| \leq \mathrm{r}$

otherwise we get

$$
\delta\left(\left\{(\mathrm{m}, \mathrm{n}, \mathrm{k}) \in \mathbb{N}^{3}:\left|\left(\mathrm{B}_{\mathrm{mnk}}(\mathrm{f}, \mathrm{x})\right)-\mathrm{f}(\mathrm{x})\right| \geq \mathrm{r}+\varepsilon\right\}\right) \neq 0
$$
for $\varepsilon=\frac{|\mathrm{f}(\mathrm{x})-\mathrm{c}|-\mathrm{r}}{3}$.

This contradicts the fact $f(x) \in s t-$ $\operatorname{LIM}^{\mathrm{r}}\left(\mathrm{B}_{\mathrm{mnk}}(\mathrm{f}, \mathrm{x})\right)$.

(b) By the equation (3.9), we can write

$s t-\operatorname{LIM}^{r}\left(B_{m n k}(f, x)\right) \subseteq \bigcap_{c \in \Gamma_{x}} \bar{B}_{r}(c)$.

Now assume that $y \in \bigcap_{c \in \Gamma_{x}} \bar{B}_{r}(c)$. Then we have

$|y-c| \leq r$

for all $\mathrm{c} \in \Gamma_{\mathrm{x}}$, which is equivalent to $\Gamma_{\mathrm{x}} \subseteq \overline{\mathrm{B}}_{\mathrm{r}}(\mathrm{y})$, i.e.,

$\bigcap_{\mathrm{c} \in \Gamma_{\mathrm{x}}} \overline{\mathrm{B}}_{\mathrm{r}}(\mathrm{c}) \subseteq\left\{\mathrm{f}(\mathrm{x}) \in \mathbb{R}^{3}: \Gamma_{\mathrm{x}} \subseteq \overline{\mathrm{B}}_{\mathrm{r}}(\mathrm{f}(\mathrm{x}))\right\}$.

Now let $\mathrm{y} \notin \mathrm{st}-\operatorname{LIM}^{\mathrm{r}}\left(\mathrm{B}_{\mathrm{mnk}}(\mathrm{f}, \mathrm{x})\right)$. Then there exists an $\varepsilon>0$ such that

$\delta\left(\left\{(\mathrm{m}, \mathrm{n}, \mathrm{k}) \in \mathbb{N}^{3}:\left|\mathrm{f}(\mathrm{x})-\left(\mathrm{B}_{\mathrm{mnk}}(\mathrm{f}, \mathrm{y})\right)\right| \geq \mathrm{r}+\varepsilon\right\}\right) \neq 0$ 
the existence of a statistical cluster point $\mathrm{c}$ of the triple sequence space of Bernstein polynomials

$\left(B_{\mathrm{mnk}}(\mathrm{f}, \mathrm{x})\right)$ with $\left|\left(\mathrm{B}_{\mathrm{mnk}}(\mathrm{f}, \mathrm{y})\right)-\mathrm{c}\right| \geq \mathrm{r}+\varepsilon$,

i.e.,

$\Gamma_{\mathrm{x}} \mathrm{U} \overline{\mathrm{B}}_{\mathrm{r}}(\mathrm{y})$ and $\mathrm{y} \notin\left\{\mathrm{f}(\mathrm{x}) \in \mathbb{R}^{3}: \Gamma_{\mathrm{x}} \subseteq \overline{\mathrm{B}}_{\mathrm{r}}(\mathrm{f}(\mathrm{x}))\right\}$.

Hence $y \in s t-\operatorname{LIM}^{r}\left(B_{\text {mnk }}(f, x)\right)$ follows from $y \in$ $\left\{\mathrm{f}(\mathrm{x}) \in \mathbb{R}^{3}: \Gamma_{\mathrm{x}} \subseteq \overline{\mathrm{B}}_{\mathrm{r}}(\mathrm{f}(\mathrm{x}))\right\}$, i.e.,

$\left\{\mathrm{f}(\mathrm{x}) \in \mathbb{R}^{3}: \Gamma_{\mathrm{x}} \subseteq \overline{\mathrm{B}}_{\mathrm{r}}(\mathrm{f}(\mathrm{x}))\right\} \subseteq \mathrm{st}-\operatorname{LIM}^{\mathrm{r}}\left(\mathrm{B}_{\mathrm{mnk}}(\mathrm{f}, \mathrm{x})\right)$.

Therefore the inclusions (3.11)-(3.13) ensure that (3.10) holds.

\section{Conclusion}

In recent years the statistical convergence has been adapted to the sequences of fuzzy numbers, interval numbers etc. In this paper we have studied the notion of Bernstein operator of rough statistical convergence of triple sequence. We define the set of Bernstein operator of rough statistical limit points of a triple sequence spaces and obtain Bernstein operator of statistical convergence criteria associated with this set. We proved some theorems of the introduced sequence spaces. This notion can be used for further generalization of such sequence spaces.

\section{References}

Aytar S (2008). Rough statistical Convergence. Numerical Functional Analysis Optimization, 29(3): 291-303.

Debnath S, Sarma B, and Das BC (2015). Some generalized triple sequence spaces of real numbers. Journal of Nonlinear Analysis and Optimization, 6(1): 71-79.

Dutta AJ, Esi A, and Tripathy BC (2013). Statistically convergent triple sequence spaces defined by Orlicz function. Journal of Mathematical Analysis, 4(2): 16-22.
Esi A (2014). On some triple almost lacunary sequence spaces defined by Orlicz functions. Research and Reviews: Discrete Mathematical Structures, 1(2): 16-25.

Esi A and Catalbas MN (2014). Almost convergence of triple sequences. Global Journal of Mathematical Analysis, 2(1): 610.

Esi A and Savas E (2015). On lacunary statistically convergent triple sequences in probabilistic normed space. Applied Mathematics \& Information Sciences, 9(5): 2529-2534.

Esi A, Araci S, and Acikgoz M (2016). Statistical Convergence of Bernstein Operators. Applied Mathematics \& Information Sciences, 10(6): 2083-2086.

Fast H (1951). Sur la convergence statistique. Colloquium Mathematicum, 2: 241-244.

Pal SK, Chandra D, and Dutta S (2013). Rough ideal convergence. Hacettepe Journal of Mathematics and Statistics, 42(6): 633640.

Phu HX (2001). Rough convergence in normed linear spaces. Numerical Functional Analysis Optimization, 22(1-2): 201224.

Sahiner A and Tripathy BC (2008). Some I related properties of triple sequences. Selcuk Journal of Applied Mathematics, 9(2): 9-18.

Sahiner A, Gurdal M, and Duden FK (2007). Triple sequences and their statistical convergence. Selcuk Journal of Applied Mathematics, 8(2): 49-55.

Steinhaus H (1951). Sur la convergence ordinaire et la convergence asymptotique. Colloquium Mathematicum, 2: 7384.

Subramanian N and Esi A (2015). The generalized tripled difference of $\chi^{3}$ sequence spaces. Global Journal of Mathematical Analysis, 3 (2): 54-60.

Tripathy BC and Goswami R (2014). On triple difference sequences of real numbers in probabilistic normed spaces. Proyecciones Journal of Mathematics, 33(2): 157-174.

Tripathy BC and Goswami R (2015a). Vector valued multiple sequences defined by Orlicz functions. Boletim da Sociedade Pranaensede Matematica, 33(1): 67-79.

Tripathy BC and Goswami R (2015b). Multiple sequences in probabilistic normed spaces. Afrika Matematika, 26(5-6): 753760.

Tripathy BC and Goswami R (2015c). Fuzzy real valued pabsolitely summable multiple sequences in probabilistic normed spaces. Afrika Matematika, 26(7-8): 1281-1289. 